\title{
Note on the Electric Splitting of Drift Shells
}

\author{
M. G. Kivelson \\ Institute of Geophysics and Planetary Physics, University of California \\ Los Angeles, California 90024

\section{J. SouThwood}

Physics Department, Imperial College of Science and Technology. London, England

\begin{abstract}
The deviation of a particle from its dipole magnetic drift shell in the presence of an electric field is reexamined. Previously published solutions are generalized to include the contribution of the corotation electric field and are extended to the case of strong drift shell splitting. Some limiting values of orbital asymmetry are obtained from an approximate form of the high-energy Allvén layer.
\end{abstract}

\section{INTRODUCTION}

In a magnetospheric convection electric field, particles of different pitch angles drifting adiabatically do not remain on the same magnetic shell as they move around the earth. Roederer and Schulz [1971], Schulz [1972], and Stern [1971] have termed this electric shell splitting. In this note we show that the previous results can be obtained from a different and simpler approach which relates the asymmetry of particle orbits to the bounce-averaged drift velocity of the particles. This formulation emphasizes the physical statement that an imposed electric field is most effective in perturbing the motion of particles which drift most slowly through it. By use of a wellknown fit to the drift velocity the coupled differential equations [Schulz, 1972; Stern, 1971] are solved to give quantitative (approximate) expressions for the dependence of drift orbits on particle pitch angle. In the first section we rederive the 'weak shell splitting' solution of Schulz [1972], and we introduce an alternative definition of weak shell splitting in which the corotation drift velocity need not be small with respect to the azimuthal magnetic drift velocity. The result that we obtain for this case is implicit in previous work [Schulz, 1972; Stern, 1971]. In the remaining sections we obtain approximate but explicit solutions for the asymmetry of drift shells in the 'strong shell splitting' case, i.e., for the case in which the cross-magnetosphere potential drop is not small with respect to the particle kinetic energy, and we discuss the limits on maximum drift shell asymmetry. The treatment here is nonrelativistic, though relativistic generalizations are indicated, and we confine our attention to a dipole magnetic field model.

\section{Small Amplitude Drift Shell Splitting}

In any axisymmetric magnetic field a coordinate $L$ may be defined which labels magnetic shells, and in particular, we can choose $L$ to be the radial distarice from the symmetry axis to the equatorial point of a field line. In this paper, $L$ is measured in units of earth radii $R_{E}$. As a particle moves across $L$ shells adiabatically, that is, conserving its magnetic moment $\mu$ and its second adiabatic invariant $J$, its change in energy $W$ with change in position is given by

$$
\left.\frac{\partial W}{\partial L}\right|_{\mu, J}=q \bar{\omega}_{d} B_{e q} R_{E}{ }^{2} L
$$

where $B_{\text {eq }}$ is the magnetic field strength at the equatorial point

Copyright $@ 1975$ by the American Geophysical Union. of the field line and $\tilde{\omega}_{d d}$ is the bounce average of the magnetic longitudinal angular drift velocity. A proof of (1) can be found in a paper by Northrop and Teller [1960] and is also given by Kivelson and Southwood [1975b]. Related forms can be found in papers by Dungey [1966], Southwood et al. [1969], and Southwood [1972]. In particular, substituting the approximate expression of Hamlin et al. [1961] for $\tilde{\omega}_{d}$ in a dipole field gives

$$
\left.\frac{\partial W}{\partial L}\right|_{\mu, J}=-\left(2.1+0.9 \sin \alpha_{\mathrm{eq}}\right) \frac{W}{L}
$$

To see the power of this approximation in shell splitting analysis, consider a particle drifting from midnight under the influence of a cross-tail uniform convection field $E$. Its change in energy is given by

$$
W-W_{M}=-q E L R_{E} \sin \phi
$$

where $\phi$ is longitude measured eastward from midnight and subscript $M$ indicates midnight. From (1), if the change in energy is small, we have the change in $L$ given by

$$
\begin{aligned}
& L-L_{M}=\left.\frac{\partial L}{\partial W}\right|_{\mu, J}\left(W-W_{M}\right) \\
& L-L_{M}=-\frac{E \sin \phi}{B_{\mathrm{eq}} \tilde{\omega}_{d} R_{E}}
\end{aligned}
$$

or using (2), we have

$$
L-L_{M}=\frac{q E \sin \phi}{W} L^{2} R_{E}\left(2.1+0.9 \sin \alpha_{\mathrm{eq}}\right)^{-1}
$$

where $E$ is measured in $\mathrm{kV} / R_{E}, W$ in $\mathrm{keV}$, and $L$ in $R_{E}$. That the shell splitting given in (5) is the same as Schulz's [1972] equation (10), which is also evaluated for $|\delta L| / L<<1$ in the nonrelativistic limit, is seen from the following argument. The necessáry derivative can be expressed as

$$
\left.\frac{\partial L}{\partial W}\right|_{\mu, J}=-\left.\frac{\partial J}{\partial W}\right|_{\mu, L}\left\{\left.\frac{\partial J}{\partial L}\right|_{\mu, W}\right\}^{-1}
$$

By direct differentiation of

$$
J=\oint d s p_{\|}=\oint d s(2 m W-\mu B)^{1 / 2}
$$

where $p_{\|}$is parallel momentum and $m$ is mass, we obtain 


$$
\left.\frac{\partial J}{\partial W}\right|_{\mu, L}=\oint \frac{d s}{v_{1}}=\tau_{b}
$$

where $\tau_{b}$ is the bounce period. For a dipole field, using

$$
\partial(d s) / \partial L=d s / L
$$

we qbtain

$$
\left.\frac{\partial}{\partial L}\right|_{\mu, W}=\frac{\tau_{b}}{L}\left\{3 W-\frac{J}{2 \tau_{b}}\right\}
$$

(correction to the form given by Dungey [1966]). From (4) and (7)-(9) it follows that

$$
\delta L=\frac{q E L^{2} R_{E} \sin \phi}{3 W-J /\left(2 \tau_{b}\right)}
$$

Noting that Schulz [1972] defines $Y$ and $T$ by

$$
J=2 m v L Y\left(\sin \alpha_{\mathrm{eq}}\right) \quad \tau_{b}=4 L v^{-1} T\left(\sin \alpha_{\mathrm{eq}}\right)
$$

we obtain the form of Schulz's equation:

$$
\delta L=2 q E L^{2} R_{E} \sin \phi W^{-1}(6-Y / T)^{-1}
$$

A simple physical interpretation of drift shell splitting follows from the form given in (5). The size of the effect is inversely proportional to the magnetic drift speed simply because the faster a particle moves across the night side the less time the convection field has to move the particle inward. Similarly, the faster a particle moves round the day side the less time there is for the convection field to move the particle sunward. In a purely dipolar magnetic field the orbits are therefore most asymmetric at small pitch angles where $\tilde{\omega}_{d}$ is smallest (see equation (6)). On the other hand, in a 'taillike' but still cylindrically symmetrical field, which may more appropriately describe the magnetic field of Jupiter beyond $20 R_{J}$ (see paper by $O p p$ [1974] and the papers following his), the large curvature of the field lines could produce angular drift velocities which decrease with increasing pitch angles near $90^{\circ}$. The corresponding asymmetry would be larger for $90^{\circ}$ pitch angle particles than for particles mirroring off the equator.

The simple approach above can be extended to include the effect of corotation electric fields. Equation (3), again in the small amplitude limit, is simply modified to

$$
W-W_{M}=-q E R_{E} L \sin \phi-q \tilde{\omega}_{c} B_{\mathrm{eq}} R_{E}^{2} L\left(L-L_{M}\right)
$$

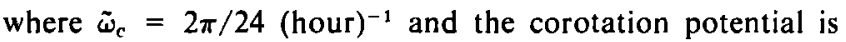
$-\tilde{\omega}_{c} B_{\mathrm{eq}} L^{2} R_{E}^{2}$, and substituting this into equation (4), we find

$$
L-L_{M}=\frac{-E \sin \phi}{B_{\mathrm{eq}} R_{E}\left(\bar{\omega}_{d}+\bar{\omega}_{c}\right)}
$$

Since $\bar{\omega}_{d}$ is positive for electrons and negative for protons, corotation enhances proton penetration of the dipole field (see, e.g., Chen [1970]).

Equation (12) may be rewritten

$$
L-L_{M}=\frac{q E \sin \phi L^{2} R_{B}}{\left(2.1+0.9 \sin \alpha_{\mathrm{eq}}\right) W-(q /|q|)(90 / L)}
$$

for terrestrial dipole field parameters, and $W$ and $q E R_{E}$ are measured in $\mathrm{keV}$.

The expression for shell splitting given in (12) is implicit in equation (9a) of Schulz [1972] (though a factor $L$ is missing in the second term of the denominator of that expression). However, Schulz used the limit $W \gg$ potential energy (from which it follows that $\left|\tilde{\omega}_{d}\right| \gg>\tilde{\omega}_{c}$ ) to define weak shell splitting.
Equations (12) and (13) follow from the alternative assumption $|\Delta W| \ll W$, which implies only that $\left|\tilde{\omega}_{d}\right| \gg[\gamma \mid L-$ $\left.\dot{L}_{M} \mid / L\right] \tilde{\omega}_{c}$. Consequently, corotation terms which are not included in Schulz's limiting expression appear in the denominators of equations (12) and (13).

\section{Strong Drift Shell Splitting}

Southwood and Kivelson [1975] have shown that the $L$ dependence of equatorial pitch angle consistent with the Hamlin et al. [1961] approximate drift velocity is

$\sin \alpha_{\text {eq }}(L)=\sin \alpha_{\text {eq } 0}\left\{1-\left(1-\sin \alpha_{\text {eq } 0}\right)\left[1-\left(L / L_{0}\right)^{0.45}\right]\right\}^{-1}$

where $\sin \alpha_{\text {eq0 }}$ is the initial pitch angle at $L=L_{0}$. From (14) it can be shown that the pitch angle dependent coefficient in (2) changes by less than $3 \%$ for $L / L_{0} \geq 0.5$. If this small variation is ignored, (2) gives an approximate relation between $W$ and $L$

$$
\frac{W}{W_{0}}=\left(\frac{L}{L_{0}}\right)^{-\gamma} \quad \gamma=2.1+0.9 \sin \alpha_{\mathrm{eq}}
$$

The simple analytic representation (15) of the pitch angle dependence of the energy gained in inward motion can be used to obtain an approximate solution for electric field shell splitting when changes in $W$ are not small with respect to $W$. For particles sufficiently energetic that corotation can be ignored, the solution is most direct. A particle of energy $W$ at any $L$ and longitude $\phi$ can be traced back to the point at which it crossed the midnight meridian where its energy $W_{M}$ is found from (3). From (15) the $L$ value at midnight is seen to be

$$
L_{M}=L\left(1+q E R_{E} L \sin \phi / W\right)^{-(1 / \gamma)}
$$

Note that the first-order term in an expansion of (16) is identical with the form previously obtained in (6), and again, the changes in $L$ are greatest for particles at small pitch angles. The variation of particle energy around the drift orbit must be found from (3).

\section{LIMITS ON THE ASYMMETRY OF DRIFT ORBITS}

The deviation from a magnetic drift shell of the orbit of a trapped particle in a magnetospheric convection field is largest for particles on an Alfvén layer, that is, on the boundary between orbits closed to drift around the earth and orbits open to the magnetopause [Alfvén and Fälthammar, 1963; Wolf, 1970; Chen, 1970]. Consequently, the properties of Alfvén layers provide upper limits on the trapped particle drift shell splitting and at the same time delimit the inward penetration of energetic particles injected from the tail by a steady convection field [e.g., Kivelson and Southwood, 1975a, b].

An Alfvén layer is defined for a particle of magnetic moment $\mu$ as a flow line on which there is a stagnation point where electric field drifts and magnetic drifts exactly balance. For particles whose energy is large with respect to their potential energy in the corotation electric field $(\sim 90 / L \mathrm{keV})$ the stagnation point on the dawn meridian for protons and on the dusk meridian for electrons is found from the approximation (15) to lie at

$$
L_{s}=\gamma W_{s} /|q| E R_{E}
$$

where $W_{s}$ is the energy at the stagnation point [Kivelson and Southwood, 1975b]. The equation for the Alfvén layer is obtained from (16) as

$$
L^{-\gamma}\left(1+q E R_{E} L \sin \phi / W\right)=L_{s}^{-\gamma}\left(1+|q| E R_{E} L_{s} / W_{s}\right)
$$

Equations (15) and (17) can be used to express this in the sim- 


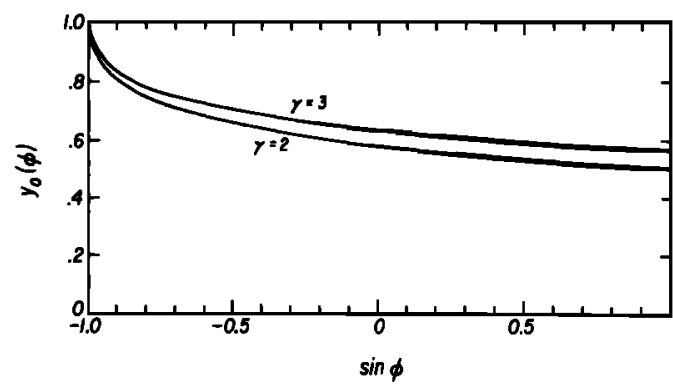

Fig. 1. Plot of $y_{0}(\phi)=L(\phi) / L_{s}$, as defined by (19), versus $\phi$ for electrons.

ple form

$$
(q /|q|) \gamma \sin \phi y_{0}^{\gamma+1}-(\gamma+1) y_{0}^{\gamma}+1=0
$$

where $y_{0}=L(\phi) / L_{s}$. For $\gamma=3,(19)$ is the equation for the Alfvén layer of equatorial particles originally given by Alfvén [Alfvén and Fälthammar, 1963]. Solutions of (19) provide estimates of the maximum asymmetry of drift orbits. A plot of the solution $y_{0}(\phi)$ for electrons is given in Figure 1. The corresponding results for energetic protons are obtained if $\phi_{p}=$ $-\phi_{e}$. In Table 1 we give some values for the maximum drift shell asymmetry for energetic particles of $0^{\circ}$ and $90^{\circ}$ pitch angles. We define $L_{M}, L_{s}$, and $L_{D}$ as the equatorial distances to the drift shell at midnight, dusk, and dawn, respectively, for electrons. For protons the local times of $L_{s}$ and $L_{D}$ are interchanged. These entries indicate that when corotation can be neglected, the electron drift orbits near dawn (or for protons near dusk) even of particles on the Alfvén layer are quite insensitive to equatorial pitch angle; i.e., the drift shell splitting is small. On the other hand, both the asymmetry of drift orbits and the dependence of the asymmetry on equatorial pitch angle become large in the direction of the stagnation point. On this side of the earth the particle energy is lowest, and convection is naturally more important. One should note that the fractional change of electron energy on an Alfven layer between midnight and dawn is of the order of $30 \%$ for $2 \leq \gamma \leq 3$

\section{SUMmary}

From the simple but useful expression given as (1) the problem of electric field drift shell splitting has been solved with minimum mathematical complexity in a manner which emphasizes the underlying physical process. The effect of corotation fields on weak drift shell splitting (equations (12) and (13)) has not been noted previously, and the approximate solutions for strong drift shell splitting (equations (15) and (16)) are also new. Upper limits to drift shell asymmetry have been obtained from considerations of the Alfven layer.

\section{Appendix: Relativistic Generalizations}

In the relativistic limit, (1) remains valid, but the bounceaveraged drift velocity must be evaluated in the high-energy limit. For a dipole field, $\left(\tilde{\omega}_{d}\right)_{\text {rel }}$ evaluated by Lew [1961] has the form

TABLE 1. Parameters of the Electron Alfvén Layer in High-Energy Limit

\begin{tabular}{cccc}
\hline & & $\left(L_{M}-L_{n}\right) /$ & $\left(L_{S}-L_{M}\right) /$ \\
$\gamma$ & $\alpha_{\text {eq }}$ & $L_{M}$ & $L_{M}$ \\
\hline 3 & $90^{\circ}$ & 0.11 & 0.59 \\
2 & $0^{\circ}$ & 0.12 & 0.75 \\
\hline
\end{tabular}

$$
\left(\bar{\omega}_{t}\right)_{\mathrm{rel}}=\left(\tilde{\omega}_{d}\right)_{\text {nonrel }} \frac{2 m_{0} c^{2}+W}{2\left(m_{0} c^{2}+W\right)}
$$

where $\left(\bar{\omega}_{d}\right)_{\text {nonrel }}$ is expressed in terms of $W$, the pitch angle dependence being the same as that in the nonrelativistic case. Consequently, (14) for $\sin \alpha_{\mathrm{eq}}(L)$ is unchanged for relativistic particles. On the other hand, the approximate form of $W(L)$ given in (15) becomes in the relativistic case

$$
\frac{W\left(2 m_{0} c^{2}+W\right)}{W_{0}\left(2 m_{0} c^{2}+W_{0}\right)}=\left(\frac{L}{L_{0}}\right)^{-\gamma}
$$

$\left(\gamma=2.1+0.9 \sin \alpha_{\text {eq }}\right)$. It is worth noting that in the limit $W$ $>>m_{0} c^{2}$, the energy change is given by $W / W_{0}=\left(L / L_{0}\right)^{-\gamma / 2}$; i.e., the fractional gain in energy for extremely energetic particles is less than that for nonrelativistic particles as they convect inward. From (A1) and (3) (unchanged for relativistic energies) the drift shell splitting follows, with the generalization of (16) simply

$L_{M}=L\left[1+\frac{2\left(W+m_{0} c^{2}\right) q E R_{E} L \sin \phi}{W\left(2 m_{0} c^{2}+W\right)}\right]^{-(1 / \gamma)}$

Acknowledgments. One of the authors (M.G.K.) wishes to thank the John Simon Guggenheim Memorial Foundation for its support and Professor J. W. Dungey for his hospitality during the tenure of her fellowship. This work is supported in part by NASA under grant NGL 05-007-004.

The Editor thanks M. Schulz and two other referees for their assistance in evaluating this paper.

\section{REFERENCES}

Alfvén, H., and C.-G. Fälthammar, Cosmical Electrodynamics, Oxford University Press, New York, 1963.

Chen, A. J., Penetration of low-energy protons deep into the magnetosphere, J. Geophys. Res., 75, 2458, 1970.

Dungey, J. W., Survey of acceleration and diffusion, in Radiation Trapped in the Earth's Magnetic Field, edited by B. M. McCormac, D. Reidel, Dordrecht, Netherlands, 1966.

Hamlin, D. A., R. Karplus, R. C. Vik, and K. H. Watson, Mirror and azimuthal drift frequencies for geomagnetically trapped particles, $J$. Geophys. Res., 66, 1, 1961.

Kivelson, M. G., and D. J. Southwood, Local time variations of particle flux produced by an electrostatic field in the magnetosphere, $J$. Geophys. Res., 80, 56, 1975a.

Kivelson, M. G., and D. J. Southwood, Approximations for the study of drift boundaries in the magnetosphere, J. Geophys. Res., 80, in press, $1975 b$.

Lew, J. S., Drift rate in a dipole field, J. Geophys. Res., 66, 2681, 1961. Northrop, T. G., and E. Teller, Stability of the adiabatic motion of charged particles in the earth's field, Phys. Rev., 117, 210-225, 1960.

Opp, A. G., Pioneer 10 mission: Summary of scientific results from the encounter with Jupiter, Science, 183, 302, 1974.

Roederer, J. G., and M. Schulz, Splitting of drift shells by the magnetospheric electric field, J. Geophys. Res., 76, 1055, 1971.

Schulz, M., Drift shell splitting at arbitrary pitch angle, J. Geophys. Res. 77, 624, 1972.

Southwood, D. J., Preservation of the second adiabatic invariant during cross $L$ diffusion, J. Geophys. Res., 77, 1123, 1972.

Southwood, D. J., and M. G. Kivelson, An approximate analytic description of plasma bulk parameters and pitch angle anisotropy under adiabatic flow in a dipolar magnetospheric field, $J$. Geophys Res., 80, 2069, 1975.

Southwood, D. J., J. W. Dungey, and R. J. Etherington, Bounce resonant interaction between pulsations and trapped particles, Planet. Space Sci., 17, 349, 1969.

Stern, D. P., Shell splitting due to electric fields, J. Geophys. Res., 76. $7787,1971$.

Wolf, R. A., Effects of ionospheric conductivity on convective flow of plasma in the magnetosphere, J. Geophys. Res., 75, 4677, 1970.

(Received July 15, 1974;

revised April 2, 1975;

accepted April 7, 1975.) 